\title{
A Robust Security Algorithm for VANETs
}

\author{
Harpreet Kaur \\ Research Scholar (ECE), \\ SBSSTC, Ferozepur
}

\author{
Amit Grover \\ Assistant Professor (ECE), \\ SBSSTC, Ferozepur
}

\begin{abstract}
The VANETs carry several security considerations. One in every of the popular and dangerous attacks is launched within the variety of Sybil, connectivity holes or cut-up attack, wherever associated in Nursing assaulter inserts a faux position inside within the cluster. The inserted faux node data is used by the hackers within the case of inconsiderate driver, traffic jams, selective collisions and different similar dangerous things. To avoid such things the VANETs should be protected against such attacks. During this paper, a completely unique answer has been projected to beat the Sybil and cut-up attacks on the VANETs. The new answer is capable of police work the faux data injections by confirmatory the VANET node behavior within the cluster. The behavior of the node includes the direction, speed and pattern. The projected model has been developed mistreatment the random waypoint model. The random waypoint model has been compared against the point of reference cluster model. The experimental results have shown the effectiveness of the projected model and also shows that RWPM is better than RPGM.
\end{abstract}

\section{Keywords}

VANET security, VANET secure mobility, connectivity hole avoidance, wormhole detection.

\section{INTRODUCTION}

Vehicular ad-hoc network (VANET) is Associate in nursing ad-hoc network that is recognized as a taxonomic group of the mobile ad-hoc network (MANAET). It is one amongst the auspicious approaches of the intelligent transit (ITS) [2]. VANET offers the no. Of options like fast changes within the constellation, high quality, repeated or periodic portioning etc. transport ad-hoc network enable inter-vehicle communication to reinforce driving expertise and road safety [12]. Communication within the transport ad-hoc network depends on the transfer of messages between many nodes within the network. It helps to reinforce the protection, driving effectiveness and relief on the course for the travellers [1] within the transport network, the messages collected from alternative nodes create use of to create the foremost of the choices.

Still, a node could perform as malicious or stingy so as to require the like alternative transport nodes. Security of the VANET has been known as an enormous challenge. The applications of the VANET compromise with life crucial information and support the period communication to try it properly, it's essential to follow some security needs like integrity, non-repudiation, authentication and privacy across assaulters and malicious attacker nodes [4]. There are a unit the no. Of attacks like part, timing, illusion, DOS [10], Sybil that not solely influences the vehicles and deriver's privacy however additionally affects the traffic safety [1][13]. In some cases, it's going to ends up in loss of life to confirm the traffic safety the VANET wants some appropriate security techniques that may assure protection across distinct misbehaviours and malicious nodes that influence the protection of the VANET [5][7].

Information distribution within the VANET takes place through the joint behaviour of the transport nodes. The messages that area unit broadcast hold the essential info like road condition, holdup, inclemency condition, emergency break events and accidents notifications etc. In this case, once a vehicle interfere or alter the messages then the results are going to be terribly risky. Hence, actus reus within the VANET is extremely necessary concern. Normally, the actus reus indicates the abnormal behaviour. Thus, the detection of the misdeed and malicious nodes includes a misconducts i.e. greatly crucial plenty of labour has been lugged dead set determine the actus reus and malicious node within the transport ad-hoc network. Generally, the actus reus detection methods may be of 2 types: knowledge central and node central actus reus detection strategy [6] [8][9][11].

The data-centric theme examines the info broadcasts between the nodes to spot the actus reus. It is fascinated by relationship among messages instead of the identities of single node. The data distributed by the nodes within the network is examined and compared with the data collected by alternative nodes. Hence, any node within the transport network that transmits some false info concerning the many events within the VANETs like faux traffic messages, false location, faux emergency events, road conditions, accidents etc. is recognized to be as misbehaving. This type of behaviour is set through knowledge central actus reus schemes [1] [3].

Non-centric techniques area unit accustomed characterize between the nodes victimization authentication. Digital signatures, security credentials area unit accustomed validate the node that transmittal the messages [3]. Such techniques area unit specialize in the node that transmittal the messages instead of the inforamtion transmits. Non-centric schemes may be any classified as activity and trust based mostly noncentric schemes. Activity techniques works by watching the node's behaviour by uses a metric helps to look at the however expeditiously a node works. Trust based mostly node-centric techniques area unit accustomed choose the nodes by its behaviour within the past and gift. This behaviour additionally accustomed access the habitual behaviour within the future [9].

Transport ad-hoc network have achieved plenty of concentration because it will improbably enhance the protection on the roads and therefore the driving conditions. Discovering the actus reus within the VANET is extremely necessary because it may be dangerous. The node central and knowledge central actus reus detection techniques have a no. of problems that demands to eliminate to create the VANET additional reliable and safe. The non-centric techniques may be increased by selecting the node as observer when acceptable validation. These techniques would like nice observations to search out the abnormal actus reus [6][8][3] Hence, to try this there's nice would like of high speed 
computation and process hardware on the on board unit (OBU) to create the choices earlier and properly. Some actus reus detection techniques use the results of short term actus reus. In knowledge central schemes, the actus reus is detected by victimization the protection alert messages, beacons etc. To decrease the overburden associated within the communication of the messages. It's been seen that nobody actus reus find theme will detect all kinds of the actus reus expeditiously within the VANETs.

\section{LITERATURE SURVEY}

S. RoselinMary [1] An Attacked Packet Detection Algorithm (APDA) which is used to detect the DOS (Denial-of- Service) attacks before the verification time. This minimizes the overhead delay for processing and enhances the security in VANET. APDA algorithm is used to improve the security of VANET system and to avoid the delay overhead in early time. The algorithm can be applied before the verification time delay overhead is minimized and will enhance the security of VANET. In future we are going to apply this algorithm for multiple invalid request send from multiple vehicles at the same time and detect the attacks in the early manner.

Daeinabi, A[2] the Detection of Malicious Vehicles (DMV) algorithm through monitoring to detect malicious nodes that drop or duplicate received packets and to isolate them from honest vehicles, where each vehicle is monitored by some of it trustier neighbors called verifier nodes. If a verifier vehicle observes an abnormal behavior from vehicle $V$, it increases distrust value of vehicle $V$. The ID of vehicle $V$ is then reported to its relevant Certificate Authority (CA) as a malicious node when its distrust value is higher than a threshold value. Performance evaluation shows that DMV can detect most existence abnormal and malicious vehicles even at high speeds.

Sanjay Silakari [3] detecting misbehavior in VANET is very crucial and indispensible as it might have disastrous consequences. This paper presents a detailed survey on some of the important research works proposed on detecting misbehavior and malicious nodes in VANETs. In addition to the details about the techniques used for misbehavior detection, nature of misbehavior, this paper categorizes the schemes for better understanding and also outlines several research scopes to make VANET more reliable and secure.

Constantinos Kolias [4] it provides a comprehensive taxonomy of attacks and countermeasures on 802.16. Each attack is classified based on several factors, e.g. its type, likelihood of occurrence, impact upon the system etc. and its potential is reviewed with reference to the standard. Possible countermeasures and remedies proposed for each category of attacks are also discussed to assess their effectiveness. Second, a full-scale assessment study of indicative attacks that belong to broader attack classes is conducted in an effort to better comprehend their impact on the 802.16 realm. As far as we are aware of, this is the first time an exhaustive and detailed survey of this kind is attempted.

Vulimiri et al. [5] have advised a probabilistic wrongdoing detection approach that is depend on the secondary data. These alerts square measure build correspondence to primary alerts. The secondary alerts square measure accustomed validate the reality and conjointly the falsity of the first alerts that square measure accessed by the transport nodes. Generally, the secondary data accepts within the variety of alerts. These alerts square measure assembled to come up with a degree of trust for the first messages.
Ghosh,M[6] A analyze (via simulations) the performance of a Misbehavior Detection Scheme (MDS) for Post Crash Notification (PCN) application. This paper observe that the performance of this proposed scheme is not very sensitive to the exact dynamics of the vehicle on small scales, so that slight error in estimating the dynamics of the detecting vehicle does not degrade the performance of the MDS.

Kim and bae [7] have advised a unique wrongdoing based mostly name management theme (MBRMS). It contains the 3 elements (a) wrongdoing detection (b) event transmit (c) international eviction algorithms to find and filter the false data within the transport networks. Each transport node manages the data system of the events and equivalent actions for determinant of misbehaving node. This system uses the outlier detection theme. MBRMS expeditiously discovers and ejects the misbehaving node

Ghosh et al. [8] planned a robust and powerful theme to work out the malicious vehicles for post crash notification application. Firstly, it acknowledges the driver's actions establishing a crash alert message. Examined quality and expected mechanical phenomenon of the transport node for the crash quality model is computed. If the distinction among 2 surpass the brink price then the alert is taken to be false. this system expeditiously decreases the false positions and therefore the false negatives whereas expeditiously discovering the wrongdoing.

Ms. Poonam Barua[9] This paper focus on security requirements, security schemes, threats, attacks and their countermeasures that provide protection from those attacks most of the security schemes are based on specific network models and complete security model for all layers is not at all present although, in future, the security scheme might become well established for individual layer.

Marco Tiloca [10] a self-adaptive and decentralized MAClayer solution against selective jamming in TDMA-based WSNs. SAD-SJ does not need a central entity, requires sensor nodes to rely only on local information, and allows them to join and leave the network without hindering other nodes activity. We show that SAD-SJ introduces a limited overhead, in terms of computation, communication and energy consumption.

Bibhu, V [11] This present the performance analysis of the black hole attack in Vehicular Ad Hoc Network. We elaborate the different types of attacks and their depth in ad hoc network. The performance metric is taken for the evaluation of attack which depends on a packet end to end delay, network throughput and network load. The delay, throughput and load are simulated by the help of OPNET 14.5 modeler. The simulation setup comprises of 30 Vehicular nodes moving with constant speed of 10 meter per second. The data rate of Vehicular nodes is $11 \mathrm{Mbps}$ with default transmitting power of 0.005 watts. With On Demand Distance Vector Routing and Optimized Link State Routing the malicious node buffer size is lowered to a level which increase packet drops.

Joe, M. M.[12] This paper, modeled network communication between vehicles and also we brought out network communication between vehicles and mobile phones. We studied the comprehensive characteristics of vehicles moving on the road and Bluetooth technology. We form the network communication among vehicles using Bluetooth technology. In order to provide securable communication between vehicles and prevent our network architecture from the hackers, we have provided securable communication between 
vehicles by authenticating the vehicles in a securable manner. Our authentication process takes place by matching the master key and slave key shared by the vehicles. We have also provided background authentication mechanism to reduce the authentication delay. Our mechanism works well and it is evaluated by the metrics provided.

Sonali Swetapadma Sahu[13] The flood of incoming messages to the target system essentially forces it to shut down, thereby denying service to the system to legitimate users. Not much research work has been done in DDoS in WSN.We are conducting a review on DDoS attack to show its impact on networks and to present various defensive, detection and preventive measures adopted by researchers till now.

\section{EXPERIMENTAL DESIGN}

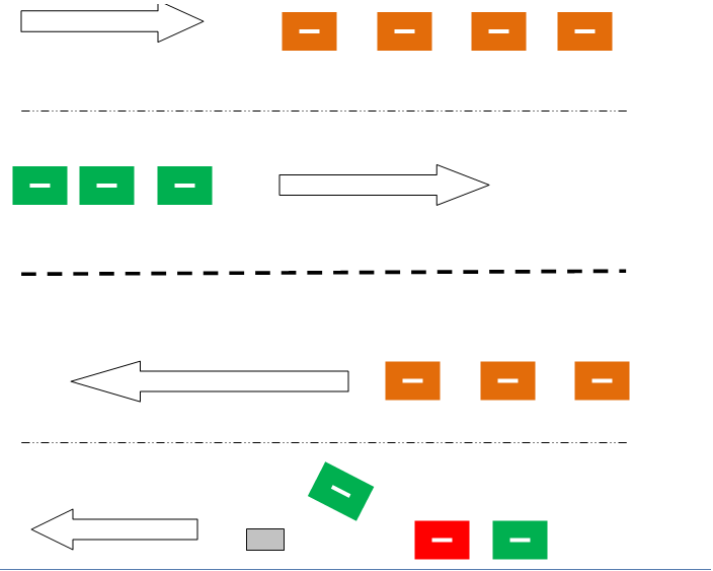

Figure 3.1: The demonstration of the prankster or Sybil attacks with single node

The on top of figure describes the traffic within the 2 directions. Every direction has 2 lanes the vehicles within the 1st lane area unit marked with inexperienced and second lane vehicles are marked with the orange colour. The red coloured vehicle within the bottom lane has been outlined because the offender node, that inject the false data within the cluster to launch the cut-up attack to require the advantage by creating its manner clear so as to facilitate the effort free movement by forcefully amending the driving direction or lane of the opposite vehicles within the cluster. The red node have planned the grey coloured Sybil node within the front of the inexperienced vehicle and have over-involved the speed, that forced the inexperienced vehicle to vary its lane to get the obstacle free movement, that directly provides the thanks to the red vehicle within the quick lane. This paper analysis, worked upon the VANET security issue of cut-up attack. During this attack one to a lot of VANET nodes propagates their false location and direction to different nodes within the cluster, which can cause accident or tie up. This assaultive mechanism may be used by terrorist or egoistic driver to mandate their intensions. During this paper, the answer recommended is applicable to the VANET cluster with none ancient setup of Road aspect Units. The planned answer is used by the VANET nodes severally or within the cluster.

Assumptions: a. All VANET nodes should bear in mind regarding
its own location and direction of movement.

b. VANET nodes is also a part of VANET cluster. c. VANET nodes should have process power their own. (Nodes shouldn't depend upon change node for the choice logic).

d. Nodes should be capable of sharing its data in step with different nodes within the neighborhood or cluster.

Work Flow:

1. Prankster node $\mathrm{P}$ send its location data $(\mathrm{X}, \mathrm{Y}$ co-ordinates) to node A

2. Node A receive the situation coordinates $\mathrm{X}$ and $\mathrm{Y}$ of node $\mathrm{P}$

3. Node A assumes its own location coordinates $\mathrm{X}$ and $\mathrm{Y}$ as central purpose i.e. Xc and $\mathrm{Yc}$.

4. Now it'll assume the situation coordinates by node $\mathrm{P}$ as $\mathrm{Xp}$ and $\mathrm{Yp}$.

5. In this simulation, every node's transmission radius is of 250 meters.

I.e. $r=250$

6. It can perform the pure mathematics formulas to seek out the situation of the purpose during a circle with the formula

a) $(\mathrm{Xp}-\mathrm{Xc})^{\wedge} 2+(\mathrm{Yp}-\mathrm{Yc})^{\wedge} 2<\mathrm{r}^{\wedge} 2$ if equation (1) is happy then the purpose is inside the circle.

b) $(\mathrm{Xp}-\mathrm{Xc})^{\wedge} 2+(\mathrm{Yp}-\mathrm{Yc})^{\wedge} 2=\mathrm{r}^{\wedge} 2$ If equation (3) is happy then the purpose is on the circle boundary

c) $(\mathrm{Xp}-\mathrm{Xc})^{\wedge} 2+(\mathrm{Yp}-\mathrm{Yc})^{\wedge} 2>\mathrm{r}^{\wedge} 2 \quad$ (4) If equation (4) is happy then the purpose is out of the circle.

7. If a degree is inside circle then, direction is calculated.

a. a. If Xp \&lt; Yp (5) then perform (N$\mathrm{y}+\mathrm{x})$ and come counter Distance wherever $\mathrm{N}$ is that the purpose on circumference.

b. Otherwise perform (x-y) and come counter Distance

c. If counter Distance is a smaller amount than $N / 2$, direction is counter clockwise

d. Otherwise direction is clockwise.

For the other tracking in the proposed model the researcher utilizing the intelligent pushback mechanism. The intelligent push back mechanism has been designed to achieve the various objectives. Proposed model has been conjointly designed for the smarter routing resolution for the conveyance ad-hoc networks using the mixture of the smart routing, with pushback mechanism for node's accessibility standing check and game suppositious approach for the aim of load balance across the most effective methods given by the routing theme. The projected model has been designed to empower the conveyance ad-hoc networks with the elongated network lifespan. The projected model has been aimed toward up the network lifespan by up knowledge the info the information turnout and by minimizing the delay and packet loss by mistreatment the energy balanced data propagation between the VANET nodes. The aimed targets are achieved by 
combining the fore techniques within the excellent sequence so as to make the proper VANET network resolution.

The pushback mechanism has been designed to examine the provision of the VANET node, wherever the information is sent from the supply node. The pushback algorithmic rule has been designed to examine the node accessibility within the terms of existence of the pushback agent on the supply and destination node, the property standing of successive hop node and therefore the standing of ingress and egress queues on successive hop node. The pushback mechanism has been designed by implementing the pushback agents to modify the pushback algorithmic rule on the node. The pushback algorithmic rule is being employed to examine the provision of the node. The property is checked mistreatment the subsequent methodology of exchange transmission:

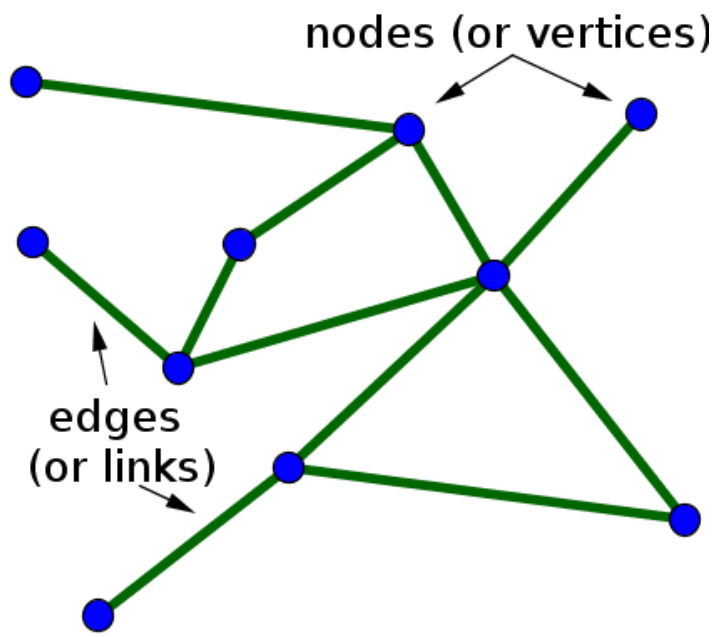

Figure 3.2: The mathematical interpretation of nodes in the form of graph [we interpret the networks as graphs in the mathematics

For the unstructured network product of $\mathrm{N}$ nodes with partial mesh property, the network nodes are drawn by the $\mathrm{NxN}$ property matrix. Just in case the network is directed, the alternative conventions are therefore the definition of the closeness matrix. During this analysis, the researcher used the basics of the closeness matrix (connectivity matrix) to style the pushback mechanism to examine the provision of the nodes. The closeness matrix may be a matrix of ones and zeros wherever one indicates the presence of association. Therefore they define A by

if there is an edge from node $j$ to node $i$

$$
A i j=1
$$

Otherwise, it will say

$$
A i j=0
$$

The pushback algorithmic rule add the matter of vertices of the directed or non-directed graphs, wherever graphs are treated because the full or partial mesh severally. The instance below shows the property of ten nodes in kind of partial mesh (partially directed graph). This analysis tagged the approachable nodes or on the market nodes love the parts of the closeness matrix:

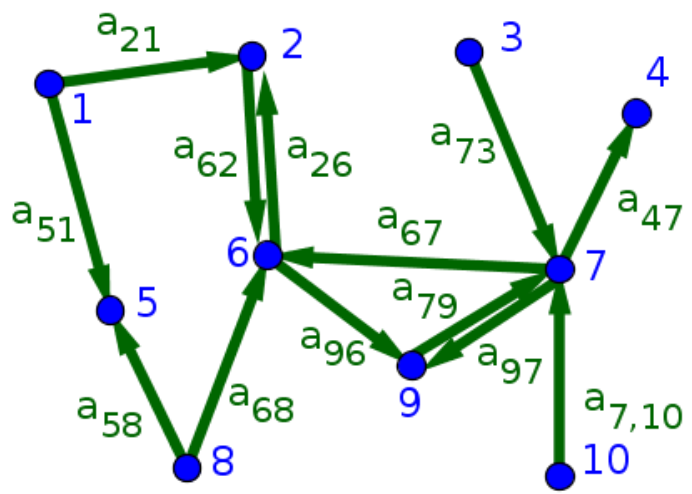

Figure 3.3: The partially directed graph with the marked directions connected with the pushback agents in the adjacent formation of nodes

The pushback Availability Relationship :- The pushback accessibility relationship between the 2 nodes has been outlined by the pushback algorithmic rule within the projected model. The provision relationship between the 2 nodes is outlined as following:

$A$ is the probability that the system will be up (its availability).

$F$ is the probability that the system will be down.

$a$ is the availability of a node.

$\mathbf{n}$ is the number of nodes in the system.

$s$ is the number of spare nodes in the system.

$f$ is the number of ways that all of the spares plus one other node can fail (that is, the number of node failures that will cause the system to fail).

Clearly, $\mathrm{A}=1-\mathrm{F}$.

At first, they consider the active/active pushback on two nodes in order to know the connection between two nodes. The single node availability is denotes as a. The probability of the availability of the node goes up with the active pushback agent. This is the probability $\mathrm{F}$ for the failure of the system:

$$
\mathbf{F}=(\mathbf{1 - a})^{2}
$$

Thus, the availability of the system, A, is

$$
A=1-F=1-(1-a)^{2}
$$

\section{RESULT ANALYSIS}

\subsection{End to end delay}

One of the comparative parameters for the comparative analysis between the RW and RPGM model is end-to-end delay. The end-to-end delay is the important network parameter and defines the performance in terms of time taken for packet delivery from source to destination.

Table 4.1: Comparative table of End-to-End delay

\begin{tabular}{|c|c|c|}
\hline No. of Nodes & RPGM & RW \\
\hline 0 & 0 & 0 \\
\hline 5 & 0.7425627017 & 0.217612 \\
\hline 10 & 2.26648752 & 0.39147 \\
\hline
\end{tabular}




\begin{tabular}{|c|c|c|}
\hline 15 & 4.589073414 & 0.535152 \\
\hline 20 & 7.567845744 & 0.647418 \\
\hline 25 & 11.04929313 & 0.739394 \\
\hline 30 & 15.25528506 & 0.898753 \\
\hline 35 & 19.9786955 & 0.995277 \\
\hline 40 & 25.03862922 & 1.054015 \\
\hline
\end{tabular}

The End To End delay for the RW model has been obtained from the simulation and recorded on the basis of number of VANET nodes (Table 4.1). With respect to the number of nodes, the RW model has been recorded at 0.54 seconds delay against the RPGM delay of approx. 4.5 seconds in the case of 15 nodes and the RW model has been recorded at 1.054 seconds delay against the RPGM delay of approx. 25 seconds in the case of 40 nodes. The comparative factor shows the clear effectiveness of the RW model while mitigating the network attacks

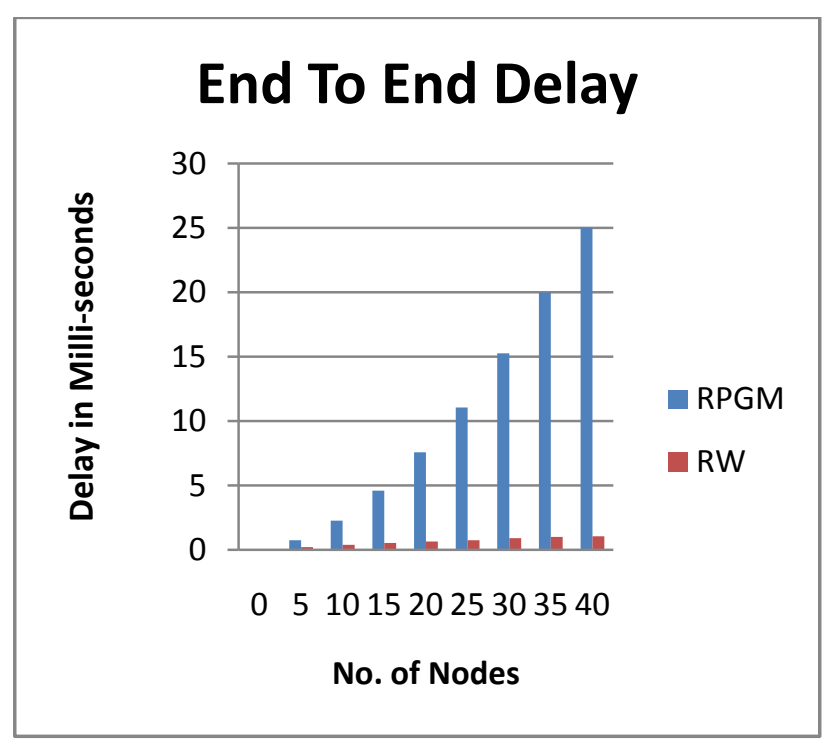

Figure 4.1: Comparison on the basis of End To End Delay

\subsection{Network load}

Network load is the parameter of the total data produced or exchanged for the processing at the given point of time in the whole VANET cluster and also given the view about resource usage.

Table 4.2: Comparative Analysis of Network Load

\begin{tabular}{|c|c|c|}
\hline No. of Nodes & RPGM & RW \\
\hline 0 & 0 & 0 \\
\hline 5 & 29.04293 & 7.866 \\
\hline 10 & 70.53971 & 9.24048 \\
\hline 15 & 119.2544 & 10.44384 \\
\hline 20 & 173.5869 & 11.06944 \\
\hline
\end{tabular}

\begin{tabular}{|r|r|r|}
\hline 25 & 231.4812 & 12.12008 \\
\hline 30 & 294.0153 & 12.94072 \\
\hline 35 & 360.5042 & 13.74664 \\
\hline 40 & 431.0778 & 14.97576 \\
\hline
\end{tabular}

The table 4.2 lists the network load during the VANET simulation of RW model and RPGM model. The RW model has been recorded with maximum load of approx $15 \mathrm{Mbps}$ against the 431 of RPGM model, which clearly indicates the improvement of fewer Loads than RPGM. The comparison has been performed on the basis of network load in the figure 4.2 .

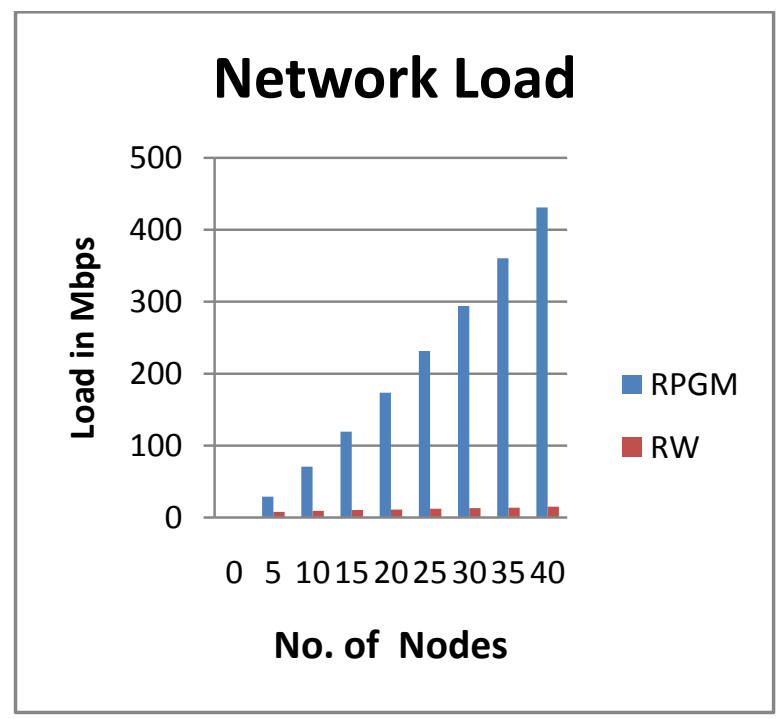

Figure 4.2: Network Load based comparative analysis

\section{Data Drop rate}

The data loss is the parameter to track the volume of the data being dropped. The data loss volumes indicate the network performance in handling the best effort packet delivery mechanism.

Table 4.3: Comparative Analysis of Data Drop Rate

\begin{tabular}{|c|c|c|}
\hline No. of Nodes & RPGM & RW \\
\hline 0 & 0 & 0 \\
\hline 5 & 35372.72 & 83108 \\
\hline 10 & 125823.5 & 156382 \\
\hline 15 & 302427.3 & 243082 \\
\hline 20 & 575271.5 & 310760 \\
\hline 25 & 922310 & 396672 \\
\hline 30 & 1370548 & 532050 \\
\hline 35 & 1904490 & 634452 \\
\hline 40 & 2526455 & 733786 \\
\hline
\end{tabular}




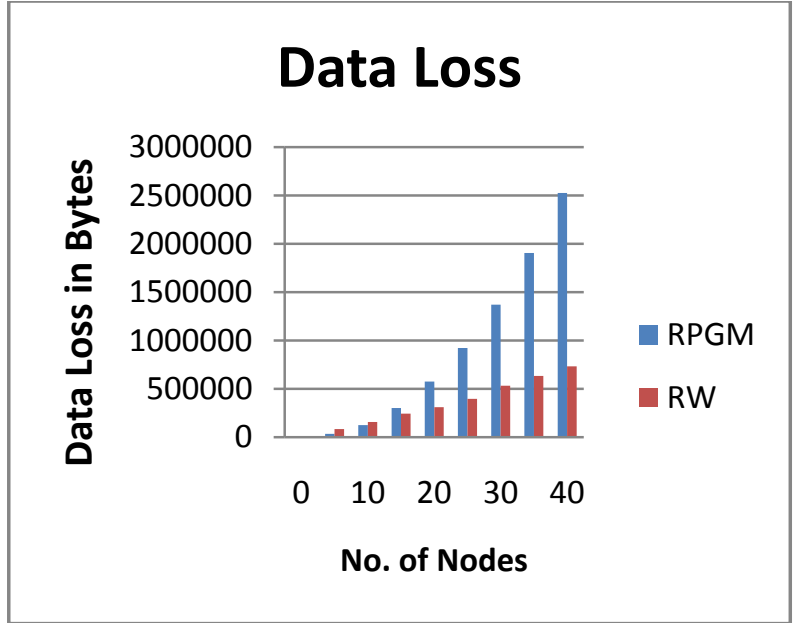

Figure 4.3: Data Loss Rate

The table 4.3 lists the data loss recorded during the RPGM and RW model simulations. The RW model has been found nearly at $0.6 \mathrm{Mbps}$ of total loss against the $1.9 \mathrm{Mbps}$ loss of the RPGM model while recorded with the 35 number of nodes.

\section{CONCLUSIONS}

The blackhole, sinkhole and connectivity hole attacks in VANET are known to significantly degrade the overall network performance and produces the greater threats to the VANET security. The VANET are known to have established in the different kinds of movement mobility models such as random way point mobility model (RWPM) and reference point group mobility model (RPGM). The nodes are guided to change their path smartly while shifting their position around the connectivity holes during the VANET mobility. The results have clearly signified the better performance of the proposed model in the RWPM than the RPGM VANET mobility model.

\section{REFERENCES}

[1] S. RoselinMary, M. Maheshwari, "Early Detection of DOS Attacks in VANET Using Attacked Packet Detection Algorithm (APDA)", vol. 1, issue 1, pp-237240 IEEE (2013).

[2] Daeinabi, A., Rahbar, A.G.: Detection of malicious vehicles (DMV) through monitoring in vehicular ad-hoc networks. Multimedia Tools Appl. vol 66(2), pp- 325338 (2013).

[3] Khan, Uzma, Shikha Agrawal, and Sanjay Silakari. "A Detailed Survey on Misbehavior Node Detection
Techniques in Vehicular Ad Hoc Networks."Information Systems Design and Intelligent Applications. vol 1, pp11-19 Springer India (2015).

[4] Constantinos Kolias, Georgios Kambourakis, Stefanos Gritzalis, "Attacks and Countermeasures on 802.16: Analysis and Assessment", CST, vol.15, issue 1, pp. 487 514, IEEE (2013).

[5] Vulimiri, A., Gupta, A., Roy, P., Muthaiah, S.N., Kherani, A.A.: Application of secondary information for misbehavior detection in VANETs. IFIP. LNCS, vol. 6091, pp. 385-396.Springer,Berlin (2010).

[6] Ghosh,M.,Varghese,A.,Kherani,A.A.,Gupta,A.: Distributed misbehavior detection in VANETs.In:Wireless Communications and Networking Conference,WCNC IEEE, pp. 1-6 (2009).

[7] Kim, C.H., Bae, and I.H.: A misbehavior based reputation management system for VANETS.LNEE vol. 181, pp- 441-450 (2012).

[8] Ghosh, M., Varghese, A., Gupta, A., Kherani, A.A., Muthaiah, S.N.: Detecting misbehaviors in VANET with integrated root-cause analysis. Ad Hoc Network.vol. 8,issue 7, pp-778-790 Elsevier (2010).

[9] Ms. Poonam Barua, Mr. Sanjeev Indora, "Overview of Security Threats in WSN", vol. 2, issue 7, pp. 422- 426, IJCSMC( 2013).

[10] Marco Tiloca, Domenico De Guglielmo, Gianluca Dini and Giuseppe Anastasi, "SAD-SJ: a Self-Adaptive Decentralized solution against Selective Jamming attack in Wireless Sensor Networks", ETFA, vol. 1, pp. 1-8, IEEE ( 2013)

[11] Bibhu, V., Roshan, K., Singh, K. B., \& Singh, D. K. (2012). Performance Analysis of black hole attack in VANET. International Journal of Computer Network and Information Security(IJCNIS),4(11),pp-47-54 (2012).

[12] Joe, M. M., Shaji, R. S., \& Kumar, K. A. (2013). Establishing Inter Vehicle Wireless Communication in Vanet and Preventing It from Hackers. International Journal of Computer Network and Information Security (IJCNIS), 5(8), pp- 55-61 (2013).

[13] Sonali Swetapadma Sahu, Manjusha Pandey, "Distributed Denial of Service Attacks: A Review", vol. 1, pp. 65-71, IJMECS (2014). 